\title{
Das Sozialgesetzbuch von I und XII - Kommentare für Lehre und Praxis
}

Utz Krahmer (Hg.): Sozialgesetzbuch I: Allgemeiner Teil. Lehr- und Praxiskommentar. 2. Auflage. Nomos Verlagsgesellschaft mbH \& Co.KG, Baden-Baden 2007. 456 Seiten. 69,- Euro. ISBN 978-38329-2221-4.

Das SGB I legt die Grundsätze der einzelnen Sozialleistungen, die Aufgaben der Leistungsträger und insbesondere das allgemeine Leistungsrecht für das gesamte Sozialrecht fest. Jede Interpretation in den besonderen Sozialleistungsbereichen der SGB-Kodifikationen greift auf diese Grundsätze zu. Die Neuauflage des Lehrund Praxiskommentars von SGB I

- legt einen Schwerpunkt auf die Leistungen der Grundsicherung für Arbeitsuchende sowie auf die Sozialhilfe und erläutert die praxisrelevanten Bezüge $\mathrm{zu}$ SGB II und XII

- erweitert insbesondere die Darstellung der richtigen Erbringung der Sozialleistungen u. a. durch die Freie Wohlfahrtspflege $(\mathbb{S 1 7})$ und

- bezieht die Themen europäische Grundrechte, europäisches Wettbewerbs- und Beihilfenrecht sowie europäisches Sozialrecht in die Erläuterungen mit ein.

In allen Bereichen auf dem neuesten Stand ist der Lehr- und Praxiskommentar von SGB I ein Muss für die Mitarbeiter bei den Leistungsträgern und Behörden, die Richter in den Sozial- und Verwaltungsgerichten, die Anwaltschaft und für die Ausbildung.

Das Autorenteam setzt sich aus erfahrenen Praktikern der Bereiche Rechtsprechung, Anwaltschaft und Verbände sowie Hochschullehrern zusammen: Prof. Dr. Utz Krahmer, Fachhochschule Düsseldorf; RiBVerwG Prof. Dr. Uwe-Dietmar Berlit; Prof. Dr. Karl-Jürgen Bieback, Universität Hamburg; RiSG Dr. Torsten Buser; Prof. Annemarie Diebold, Fachhochschule Ludwigsburg; Dr. ThomasPeter Gallon, Senatsverwaltung für Integration, Arbeit und Soziales, Berlin; Prof. Dr. Rainer Kessler, Fachhochschule Wiesbaden; Prof. Dr. Volker Neumann, Universität Berlin; Prof. Helmut Reinhardt, Fachhochschule Ludwigsburg; RA Ronald Richter; Prof. Dr. Klaus Rieken- brauk, Fachhochschule Düsseldorf; RiBVerwG Dr. Ralf Rothkegel; Ass. Jur. Martina Rudolph; Marie-Luise SchifferWerneburg, Diakonisches Werk der EKD e. V.; RiLSG Hinnerk Timme.

Johannes Münder (Hg.): Sozialgesetzbuch II. Grundsicherung für Arbeitsuchende. Lehr- und Praxiskommentar. 2. Auflage. Nomos Verlagsgesellschaft mbH \& Co.KG, Baden-Baden 2007. 786 Seiten. 44,- Euro. ISBN 978-3-83291783-8.

Die Grundsicherung für Arbeitsuchende ist in wesentlichen Punkten novelliert. Durch das SGB II-Änderungsgesetz und insbesondere durch das Fortentwicklungsgesetz sind weitgehende Leistungseinschnitte zum 1. August 2006 Gesetz geworden. Was bedeuten diese mit der »Optimierung des Leistungsrechts « und der »Vermeidung von Leistungsmissbrauch « begründeten Änderungen für das Regelungssystem des SGB II und die Auslegungspraxis? Die Neuauflage des Lehr- und Praxiskommentars zu SGB II

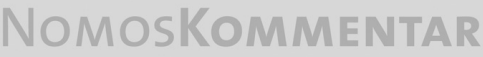

Münder | Armborst | Berlit | Bieritz-Harder | Birk | Brühl | Conradis | Geiger | Krahmer | Niewald | Roscher | Schoch

\section{Sozialgesetzbuch XII}

Sozialhilfe

Lehr- und Praxiskommentar

8. Auflage

Nomos

Im Jahre 1985 das erste Mal erschienen, hat sich der Lehr-und Praxiskommentar zur Sozialbilfe zum Klassiker gemausertund ist zum Vorbild für eine ganze Reibe von Gesetzeskommentaren geworden. gibt die praktischen Antworten und bietet eine vollständige, verständliche und aktuelle Kommentierung des novellierten SGB II, unter besonderer Berücksichtigung der Praxisauswirkung insbesondere der Leistungskürzungen. Behandelt werden dabei ausführlich die Erweiterung der Bedarfsgemeinschaft um Jugendliche, die das 25. Lebensjahr noch nicht vollendet haben, zusammen mit den dazugehörenden Beweislastfragen. Anlässlich dieser Gesetzvorgaben interpretiert der Lehrund Praxiskommentar kritisch die Verschärfung von Sanktionsmöglichkeiten. Die für die Auslegung des Gesetzes - vor allem für die Interpretation der vielfältigen unbestimmten Rechtsbegriffe - prägenden Entscheidungen der Sozialgerichte seit Inkrafttreten des SGB II sind durchgängig berücksichtigt.

Jürgen Kruse, Peter-Bernd Lüdtke, HansJoachim Reinhard, Jürgen Winkler, Irene Zamponi: Sozialgesetzbuch III. Arbeitsförderung. Lehr- und Praxiskommentar. Nomos Verlagsgesellschaft $m b H$ \& Co.KG, Baden-Baden 2008. 1.060 Seiten. 79,- Euro. ISBN 978-3-8329-0309-1.

Durch die letzten Gesetzesnovellen sind gravierende Änderungen im Arbeitsförderungsrecht in Kraft getreten, auf die sich die Beratungspraxis einstellen muss. Der Lehr- und Praxiskommentar zu SGB III bringt den Leser auf den neuesten Stand und bietet eine praxisnahe Erläuterung des novellierten Gesetzes. III. Der neue Kommentar verarbeitet zahlreiche Änderungsgesetze, unter anderem

- das Gesetz zur Verbesserung der Beschäftigungschancen älterer Menschen

- das Rentenversicherungs-Altersgrenzenanpassungsgesetz

- das Dienstrechtsanpassungsgesetz für die Bundesagentur für Arbeit

- das Gesetz zur Umsetzung aufenthaltsund asylrechtlicher Richtlinien der Europäischen Union

- das 4. Gesetz zur Änderung des SGB III

- das Gesetz zur Förderung der zusätzlichen Altersvorsorge und zur Änderung des SGB III

- das Gesetz zur Änderung des SGB IV und anderer Gesetze 
- das 6. Gesetz zur Änderung des SGB III und anderer Gesetze

- das 22. Gesetz zur Änderung des Bundesausbildungsförderungsgesetzes Der Lehr- und Praxiskommentar berükksichtigt die Neuregelungen durch das 7. SGB III-Änderungsgesetz vom 8. April 2008, insbesondere die verlängerte Bezugsdauer bei ALG I. Auch die neueste Rechtsprechung der Arbeits- und Sozialgerichte ist umfassend eingearbeitet. Das Buch ist der ideale Ratgeber für alle Praktiker im Bereich des Arbeitsförderungsrechts. Diesen Kommentar brauchen Rechtsanwälte, Richter und Mitarbeiter der Arbeitsagenturen.

Jürgen Winkler (Hg.): Sozialgesetzbuch IV. Gemeinsame Vorschriften für die Sozialversicherung. Lehr- und Praxiskommentar. Nomos Verlagsgesellschaft mbH \& Co.KG, Baden-Baden 2007. 640 Seiten. 69,- Euro. ISBN 978-3-8329-1382-3.

Das Vierte Buch des Sozialgesetzbuches enthält grundlegende Vorschriften für alle Zweige der Sozialversicherung und für das Arbeitsförderungsrecht. Diese Regelungen sind nicht nur für die Sozialversicherungsträger, sondern auch für die betriebliche Praxis von zentraler Bedeutung. Der Lehr- und Praxiskommentar bietet eine umfassende und fundierte Kommentierung des SGB IV. Er konzentriert sich vor allem auf die in der Praxis immer wiederkehrenden Hauptprobleme:

- Beschäftigung und selbstständige Tätigkeit

- Arbeitsentgelt und sonstiges Einkommen

- Leistungen und Beiträge

- Meldepflichten des Arbeitgebers

- Verfassung und Organe der Sozialversicherungsträger

- Sozialversicherungsausweis

Der Lehr- und Praxiskommentar zu SGB IV berücksichtigt zahlreiche Änderungsgesetze, insbesondere

- das Gesetz zur Umsetzung der Gleichbehandlungsrichtlinien der Europäischen Union und

- das Gesetz zur Einführung des Elterngeldes

Auch die Neuregelungen durch die Gesundheitsreform zum 1. April 2007 sind eingearbeitet. Die Autoren sind ausgewiesene Experten im Sozialrecht: RiSG Dr. Tilman Breitkreuz; RiBSG a. D. PeterBernd Lüdtke; Prof. Dr. Renate Oxenknecht-Witzsch, Katholische Universität Eichstätt-Ingolstadt; Prof. Dr. Rainer
Vor, Hochschule für Technik, Wirtschaft und Kultur Leipzig; RiLSG Dr. Roland Wietek; Prof. Dr. Jürgen Winkler, Katholische Fachhochschule Freiburg.

Jürgen Kruse, Prof. Dr. Andreas Hänlein (Hg.): Sozialgesetzbuch V. Gesetzliche Krankenversicherung. Lehr- und Praxiskommentar. 3. Auflage. Nomos Verlagsgesellschaft mbH \& Co.KG, Baden-Baden 2009. 1.548 Seiten. 99,- Euro. ISBN 978-3-8329-1381-6.

Mit Stand vom 1. Januar 2009 ergeben sich durch das Gesetz zur Weiterentwicklung der Organisationsstrukturen in der gesetzlichen Krankenversicherung (GKVOrgWG) bedeutende strukturelle Änderungen in der gesetzlichen Krankenversicherung. Die dritte Auflage des Lehr- und Praxiskommentars zum SGB V berükksichtigt die:

- Herstellung der Insolvenzfähigkeit aller Krankenkassen ab dem 1. Januar 2010,

- Neugestaltung der Finanzierung der Landesverbände nach dem Wohnortprinzip,

- Veränderungen zur Hilfsmittelversorgung

- Neuregelung des Anspruchs auf enterale Ernährung

- Aufhebung der Altersgrenze für die an der vertragsärztlichen Versorgung teilnehmenden Leistungserbringer.

Die Neuauflage stellt alle Inhalte der Gesundheitsreform und deren Finanzierung durch das GKV- Wettbewerbsstärkungsgesetz und dessen Änderungen durch das GKV-OrgWG auf dem Rechtsstand vom 1. Januar 2009 übersichtlich dar und erläutert ausführlich die neue »Versicherungspflicht für jedermann «. Einbezogen sind die Änderungen des Leistungskatalogs, der Zuzahlungsregelungen und der Wahlmöglichkeiten der Versicherten. Die Autoren erläutern zudem die Neuausrichtung der Arzneimittelversorgung unter wettbewerbsrechtlichen Gesichtspunkten und die Reform des Verhältnisses zwischen privater und gesetzlicher Krankenversicherung. Der Lehr- und Praxiskommentar zum SGB V ist das ideale Werk für Entscheidungsträger und deren Mitarbeiter in Krankenkassen, Verbänden, Gesundheitsbehörden, Krankenhausverwaltungen sowie für Ärzte, Rechtsanwälte, Richter, Ausbilder und Studierende. Autoren des Buches sind: Klaus-Peter Adelt, Justiziar a. D., Datenschutzbeauf- tragter a. D., Bochum; Dr. Christian Auktor, Notar, Fürth/Bayern; Prof. Dr. Andreas Hänlein, Universität Kassel; Tamara Henle, Rechtsanwältin, BKKLandesverband Bayern, München; Petra Kraftberger, Justiziarin, Spitzenverband Bund der Krankenkassen (GKV-Spitzenverband), Berlin; Prof. Dr. Jürgen Kruse, Evangelische Fachhochschule Nürnberg, Rechtsanwalt und Fachanwalt für Sozialrecht in München; Rita Murawski, Justiziarin, AOK Bayern, München; Corinna Roß, Rechtsanwältin, Siemens Betriebskrankenkasse, München.

Helmut Reinhardt (Hg.): Sozialgesetzbuch VI. Gesetzliche Rentenversicherung. Lehr- und Praxiskommentar. Nomos Verlagsgesellschaft $m b H$ \& Co.KG, Baden-Baden 2006. 1.161 Seiten. ISBN 978-3-8329-1355-7. (Derzeit nicht lieferbar, Neuauflage in Vorbereitung).

Die Gesetzliche Rentenversicherung ist aufgrund der zahlreichen Reformen kaum noch zu durchschauen. Der Lehrund Praxiskommentar zum SGB VI

- bereitet die Materie übersichtlich auf

- behandelt alle wichtigen Rechtsbereiche wie u. a. versicherter Personenkreis, Leistungen der gesetzlichen Rentenversicherung, Rentenberechnung, Versorgungsausgleich und

- ist auf die Ansprüche der Praxis zugeschnitten.

Die Autoren: Birgit Ehnes, Deutsche Rentenversicherung Rheinland-Pfalz; Carsten Friedrichsen, Deutsche Rentenversicherung Rheinland-Pfalz; Stefan Hirsch, Verwaltungs-Fachhochschule Wiesbaden; Michael Quinten, Deutsche Rentenversicherung Rheinland-Pfalz; Professor Helmut Reinhardt, Fachhochschule Ludwigsburg; Dietrich Schoch, Regierungsdirektor a. D.; Wolfgang Silber, Deutsche Rentenversicherung Baden-Württemberg; Bernhard Sona, Deutsche Rentenversicherung Rheinland-Pfalz; Michael Spegel, Deutsche Rentenversicherung Baden-Württemberg; Gerd-Volker Stock, Leitender Verwaltungsdirektor a. D., Deutsche Rentenversicherung Baden-Württemberg.

Edgar Franke, Thomas Molkentin (Hg.): Sozialgesetzbuch VII. Gesetzliche Unfallversicherung. Lehr- und Praxiskommentar. 2. Auflage. Nomos Verlagsgesellschaft $m b H$ \& Co.KG, Baden-Baden 2007. 820 Seiten. 98,- Euro. ISBN 978-38329-1007-5. 
Der Lehr- und Praxiskommentar zu SGB VII bietet einen umfassenden Überblick über das Recht der gesetzlichen Unfallversicherung. Die Regelungen des Unfallversicherungsrechts werden praxisnah und verständlich erläutert. Die Neuauflage wurde durchgängig aktualisiert. Sie berücksichtigt zahlreiche Änderungsgesetze und die neuste Rechtsprechung. Der benutzerfreundliche Kommentar

- konzentriert sich auf besonders praxisrelevante Bestimmungen (z. B. über den versicherten Personenkreis und die Versicherungsfälle)

- geht auch auf Fragen der Unfallverhütung und der Leistungsarten (Heilbehandlung, Rehabilitation, Renten, Abfindungen) ein und

- berücksichtigt die Belange der gewerblichen und landwirtschaftlichen Berufsgenossenschaften ebenso wie die der gesetzlichen Unfallversicherungsträger der öffentlichen Hand.

Der neue Lehr- und Praxiskommentar ist das ideale Hilfsmittel für Mitarbeiter der Unfallversicherungsträger, Betriebsund Personalräte, Rechtsanwälte und Sozialrichter. Die Autoren sind erfahrene Fachhochschullehrer und Verwaltungspraktiker: Prof. Dr. Edgar Franke, Bürgermeister der Stadt Gudensberg, Lehrbeauftragter an der Hochschule für Gesetzliche Unfallversicherung; Martin Kunze, Landesverwaltungsdirektor, Leiter der Rehabilitations- und Leistungsabteilung der Landesunfallkasse Freie und Hansestadt Hamburg; Klaus Merle, Dozent an der Fachhochschule des Bundes für öffentliche Verwaltung, Fachbereich Landwirtschaftliche Sozialversicherung; Dr. Thomas Molkentin, Leiter des Referats »Unfallversicherung " im Bundesministerium für Arbeit und Soziales; Hans-Jürgen Rapp, Fachhochschullehrer an der Hochschule der Gesetzlichen Unfallversicherung; Rainer Richter, Leiter der Rechtsabteilung, Bayerischer Gemeindeunfallversicherungsverband; Harald Streubel, Leiter der Stabstelle Grundsatzangelegenheiten und Selbstverwaltung bei der Unfallkasse des Bundes; Prof. Dr. Axel Weiß, Rechtsanwalt und Fachhochschullehrer an der Hochschule für Gesetzliche Unfallversicherung; Eberhard Ziegler, Ständiger Vertreter des Bundesverbandes der Unfallkassen im Berliner Büro der Deutschen Gesetzlichen Unfallversicherung.
Peter-Christian Kunkel (Hg.): SGB VIII. Kinder- und Jugendhilfe. Lehr- und Praxiskommentar. 3. Auflage. Nomos Verlagsgesellschaft mbH \& Co.KG, BadenBaden 2006. 1.119 Seiten. 79,- Euro. ISBN 978-3-8329-1380-9.

Der Lehr- und Praxiskommentar zum SGB VIII integriert selbstverständlich das Gesetz zur Weiterentwicklung der Kinder- und Jugendhilfe (KICK). Das Buch

- erläutert die Neuregelung der Inobhutnahme von Kindern und Jugendlichen

- legt den Schutzauftrag des Jugendamtes dar

- schafft Klarheit bei der Neuregelung der Leistungsvergabe für Kinder und Jugendliche sowie bei der Neuregelung der Kostenbeteiligung

- zeigt umfassend den verstärkten Nachrang der Jugendhilfe im Verhältnis zu den Eltern auf.

Ebenfalls eingearbeitet ist das Tagesbetreuungsausbaugesetz (TAG) zur stärkeren Förderung von Kindertageseinrichtungen und der Kindertagespflege. Somit ist der Kommentar auch allen, die für den Ausbau des Förderangebots verantwortlich sind, ein wertvolles Hilfsmittel. Der besondere Vorteil: ausführliche Darstellungen der Themen strafrechtliche Garantenhaftung, Rechtsfragen Ehrenamtlicher, Anwalt des Kindes, Wettbewerbsrecht der Europäischen Union sowie ein aktualisierter Anhang "Verfahren und Rechtsschutz«.

Dirk H. Dau, Franz Josef Düwell, Hartmut Haines (Hg.): Sozialgesetzbuch IX. Rehabilitation und Teilhabe behinderter Menschen. Lebr-und Praxiskommentar. 2. Auflage. Nomos Verlagsgesellschaft $m b H$ \& Co.KG, Baden-Baden 2009. 1.066 Seiten. 79,- Euro. ISBN 978-38329-0925-3.

Der neue Lehr- und Praxiskommentar zu SGB IX erläutert das gesamte Behindertenrecht zuverlässig und praxisorientiert. Auch die wichtigen Neuregelungen durch das Gesetz zur Einführung Unterstützter Beschäftigung vom 22. Dezember 2008 sind berücksichtigt. Die zweite Auflage verarbeitet weiterhin zahlreiche Änderungsgesetze, zuletzt

- das Gesetz zur Umsetzung der EUGleichbehandlungsrichtlinien

- das Gesetz zur Änderung des Betriebsrentengesetzes und anderer Gesetze

- das GKV-Wettbewerbsstärkungsgesetz
- das Zweite Gesetz zum Abbau bürokratischer Hemmnisse insbesondere in der mittelständischen Wirtschaft

- das Gesetz zur Änderung des Bundesversorgungsgesetzes und anderer Vorschriften des Sozialen Entschädigungsrechts.

Die Rechtsprechung der Arbeits- und Sozialgerichte ist umfassend eingearbeitet, insbesondere die jüngsten Entscheidungen des Bundesarbeitsgerichts zum Sonderkündigungsschutz für Schwerbehinderte. Die Kommentierung zu $\$ 125$ SGB IX berücksichtigt das Urteil des Europäischen Gerichtshofes vom 20. Januar 2009 zum Wegfall der Befristung des Urlaubs bei Arbeitsunfähigkeit. Der besondere Praxisvorteil: Der Anhang enthält eine kompakte Darstellung zum Verfahren und zum Rechtsschutz. Der neue Lehr- und Praxiskommentar ist der ideale Ratgeber für alle Praktiker im Bereich des Behindertenrechts. Diesen Kommentar brauchen Rechtsanwälte, Arbeits- und Sozialrichter, Rehabilitationsträger, Integrationsämter, Behinderten- und Wohlfahrtsverbände, Personalabteilungen in Unternehmen, Betriebs- und Personalräte, Gewerkschaftssekretäre und Schwerbehindertenvertreter. Autoren sind: Dirk H. Dau, Richter am Bundessozialgericht a. D., Hamburg; Berthold Deusch, Kommunalverband für Jugend und Soziales Baden-Württemberg, Stuttgart/Karlsruhe; Franz Josef Düwell, Vorsitzender Richter am Bundesarbeitsgericht, Erfurt; Dr. Hartmut Haines, Ministerialrat i. R., zuletzt im Bundesministerium für Arbeit und Soziales, Bonn; Günther Hoffmann, Rechtsanwalt und Notar, Bremen; Bernward Jacobs, Rechtsanwalt, Münster; Olaf Liebig, Regierungsdirektor im Bundesministerium für Gesundheit, Berlin.

Bjöm Diering, Hinnerk Timme, Dirk Waschull (Hg.): Sozialgesetzbuch X. Sozialverwaltungsverfahren und Sozialdatenschutz. Lehr- und Praxiskommentar. 2. Auflage. Nomos Verlagsgesellschaft mbH \& Co.KG, Baden-Baden 2007. 812 Seiten. 79,- Euro. ISBN 978-3-8329-2223-8.

Die Neuauflage präsentiert den aktuellen Stand in den Kerngebieten des sozialrechtlichen Verwaltungsverfahrens. Der Lehrund Praxiskommentar berücksichtigt

- die umfangreichen Gesetzesänderungen beim Sozialdatenschutz

- die Auswirkungen der Neuregelungen im Aufenthaltsrecht, $\mathbb{} 71$ SGB X 
- die verfahrensrechtlichen Auswirkungen durch die Änderungen im SGB II und XII.

Ein umfassender Anhang zum verwaltungsgerichtlichen Verfahren erläutert praxisnah die Umsetzung der Verfahrensprobleme im Prozess vor den Sozialgerichten. Die Autoren kommen aus den Bereichen Wissenschaft, Justiz, Anwaltschaft und Verwaltung und vereinen die notwendige Sachkompetenz für die Interpretation der Regeln des Sozialverwaltungsverfahrens: Walter Böttiger, Richter am SG Stuttgart, derzeit wissenschaftlicher Mitarbeiter am Bundessozialgericht; Dr. Tilman Breitkreuz, Richter am SG Aachen, derzeit wissenschaftlicher Mitarbeiter am Bundesverfassungsgericht; Dr. Björn Diering, Rechtsanwalt, Fachanwalt für Sozialrecht und Verwaltungsrecht; Prof. Dr. Heinrich Lang, Diplom-Sozialpädagoge, Universität Rostock; Prof. Dr. Stephan Rixen, Universität Kassel; Dr. Knut Seidel, Rechtsanwalt; Hinnerk Timme, Richter am LSG Schleswig-Holstein; Prof. Dr. Dirk Waschull, Fachhochschule Münster, Richter am LSG Nordrhein-Westfalen, ehemals Leiter des Rechts- und Versicherungsamts der Stadt Aachen.

Thomas Klie, Utz Krahmer (Hg.): Sozialgesetzbuch XI. Soziale Pflegeversicherung. Lehr- und Praxiskommentar. 3. Auflage. Nomos Verlagsgesellschaft mbH \& Co.KG, Baden-Baden 2009. 1.405 Seiten. 89,- Euro. ISBN 978-3-8329-2718-9.

Mit der "Reform zur strukturellen Weiterentwicklung der Pflegeversicherung « wurde die umfassendste Änderung der Pflegeversicherung seit ihrer Einführung vor 13 Jahren vollzogen. Die finanziellen Leistungen im Rahmen der Pflegestufen sind gestiegen, die ambulante Versorgung wird ausgeweitet und das bürgerschaftliche Engagement wird gestärkt. Die dritte Auflage des Lehr- und Praxiskommentars zu SGB XI bietet den Stand vom 1. Januar 2009 und bringt Interpretationssicherheit in den neuen Rechtsfragen. Schwerpunkte des Kommentars sind:

- der neue Anspruch auf Pflegeberatung (»Fallmanagement «)

- die Möglichkeiten und rechtlichen Grenzen der optionalen Einrichtung von Pflegestützpunkten

- die neu geregelte »Pflegezeit «

- der ausgebaute Anspruch auf Tagespflege
- die neuen umfangreichen Leistungen für demenzkranke Menschen

- die neuen Förderleistungen zur Prävention und Rehabilitation

- die neuen Möglichkeiten der privaten Pflege-Zusatzversicherung sowie

- die neu eingeführte Übertragbarkeit der individuellen Altersrückstellungen und den damit verbundenen Härteregelungen bei niedrigen Einkommen

- die neuen Qualitätssicherungsvorschriften für Heime (erweiterte Heimaufsicht, Qualitätsanforderungen, »Transparenzgebot «).

Die Neuauflage berücksichtigt zudem die neueste Rechtsprechung des Bundessozialgerichts. Sie legt Wert auf eine klare und verständliche Erläuterung, auch der praxisrelevanten Verfahrens- und Rechtschutzfragen. Das aktuelle Werk bietet auch eine Kommentierung zum Pflegezeitgesetz und berücksichtigt bereits die Neuregelungen durch das GKVOrgWG vom 15. Dezember 2008. Experten machen den Lehr- und Praxiskommentar zu SGB XI zum notwendigen Handwerkszeug für Anwaltschaft und Richter, Mitarbeiter bei den Pflegekassen, Verbänden und Behörden im Pflegebereich wie auch bei den beteiligten Sozialhilfeträgern sowie für die Ausbildung. Die Autoren: Dr. Annett Böhm, Rechtsanwältin, Fachanwältin für Arbeitsrecht, Stuttgart; Dr. Thomas-Peter Gallon, Referatsleiter, Senatsverwaltung für Integration, Arbeit und Soziales, Berlin; Dr. Sven Höfer, Rechtsanwalt, Freiburg im Breisgau; Hans-Jörg Holtbrügge, Richter am Oberverwaltungsgericht Münster; Prof. Dr. Thomas Klie, Evangelische Fachhochschule Freiburg; Prof. Dr. Utz Krahmer, Fachhochschule Düsseldorf; Dr. Bettina Leonhard, Dozentin an der HTWK Leipzig; Dr. Albrecht Philipp, Rechtsanwalt, Fachanwalt für Verwaltungsrecht und Fachanwalt für Sozialrecht, München; Dr. Markus Plantholz, Rechtsanwalt, Fachanwalt für Medizinrecht, Hamburg; Prof. Dr. Silvia Pöld-Krämer, Fachhochschule Bielefeld; Ronald Richter, Rechtsanwalt, Fachanwalt für Steuerrecht, Hamburg; Prof. Dr. Michaela Röber, Fachhochschule Frankfurt am Main; Marie-Luise Schiffer-Werneburg, Rechtsanwältin, Brandenburg; Paul Schmäing, Referatsleiter Verträge, Abteilung Pflege, VdAK/AEV e. V., Siegburg; Wolfgang Schuldzinski, Rechtsanwalt, Verbraucher-Zentrale Nordrhein-Westfalen, Düs- seldorf; Georg Vogel, Fachreferatsleiter, Techniker Krankenkasse, Hamburg.

Johannes Münder, Christian Armborst, Prof. Dr. Uwe Berlit, Renate Bieritz-Harder, Ulrich-Arthur Birk, Albrecht Brühl, Wolfgang Conradis, Udo Geiger, Albert Hofmann, Utz Krahmer, Stephan Niewald, Falk Roscher, Dietrich Schoch: Sozialgesetzbuch XII. Sozialhilfe. Lehr-und Praxiskommentar. 8. Auflage. Nomos Verlagsgesellschaft mbH \& Co.KG, Baden-Baden 2008. 1.017 Seiten. 44,- Euro. ISBN 978-3-8329-2930-5.

Drei Jahre nach Eingliederung der Sozialhilfe als Zwölftes Buch in das Sozialgesetzbuch erscheint die Neuauflage dieses Klassikers. Das Werk

- stellt die seitdem geführten rechtlichen Diskussionen sowie die umfassende aktuelle Rechtsprechung ausführlich dar und

- legt einen Schwerpunkt auf die zahlreichen Änderungen durch das Gesetz zur Änderung des Zwölften Buches Sozialgesetzbuch und anderer Gesetze vom 2.12.2006 wie u. a. die Erhöhung des Barbetrages ( $\$ 35$ Abs. 2), die Angleichung des Regelsatzes ( $\$ 28$ Abs. 2) oder den Anspruchsausschluss für Ausländer ( $\int 28$ Abs. 3). Ausführliche Anhänge zum Asylbewerberleistungsgesetz sowie zum Verfahren im SGB XII unterstützen den Praktiker im Streitfall. Der Kommentar ist bedeutsam für Leistungsberechtigte, Beraterinnen und Berater in den Wohlfahrtsverbänden, Anwaltschaft, Mitarbeiterinnen und Mitarbeiter in den Sozialverwaltungen, Richterinnen und Richter. Das Werk richtet sich darüber hinaus an Studierende und Lehrende der Hochschulen. Die Autoren: Prof. Dr. Johannes Münder, Technische Universität Berlin; MinR Christian Armborst, Niedersächsisches Ministerium für Frauen, Arbeit und Soziales; RiBVerwG Prof. Dr. Uwe Berlit; Prof. Dr. Renate Bieritz-Harder, Fachhochschule Oldenburg/Ostfriesland/Wilhelmshaven; Prof. Dr. Ulrich-Arthur Birk, Universität Bamberg; Prof. Dr. Albrecht Brühl, Fachhochschule Darmstadt; RA FASozR Dr. Wolfgang Conradis; RiSG Udo Geiger, Berlin; Prof. Dr. Utz Krahmer, Fachhochschule Düsseldorf; RiLSG Stephan Niewald, Berlin; Prof. Dr. Falk Roscher, Hochschule Esslingen; RegDir a.D. Dietrich Schoch, Ombudsmann der ARGE SGB II Duisburg. 
„Die zehn Gebote sind deswegen so kurz und logisch, weil sie ohne Mitwirkung von Juristen zustande gekommen sind."

Charles de Gaulle, französischer Politiker (1890-1970)

"Aber die Armen sind auf die Gerechtigkeit angewiesen, die Reichen sind auf die Ungerechtigkeit angewiesen, das entscheidet." Bertolt Brecht, deutscher Schriftsteller (1898-1956)

"Erfahrene Juristen bezeugen, dass es vor Gericht von Vorteil sein kann, wenn man im Recht ist."

Graham Chapman, britischer Schriftsteller (1941-1989)

"Es gibt Gerechte und Ungerechte auf dieser Welt.

Wer ungerecht ist, entscheiden die Gerechten."

Robert Lembke, deutscher Schriftsteller und Fernsehmoderator (1913-1989)

"Das Recht durchzusetzen, ist im Umfeld eines

Rechtsstaates prinzipiell möglich."

Michael Stolleis, deutscher Jurist (geb. 1941)

"Mit Gesetzen ist es wie mit Würstchen. Es ist besser, wenn man nicht sieht, wie sie gemacht werden." Otto von Bismarck, deutscher Politiker (1815-1898)

„Der Verbrecher produziert nicht nur Verbrechen, sondern auch das Kriminalrecht und damit auch den Professor, der Vorlesungen über das Kriminalrecht hält, und zudem das unvermeidliche Kompendium, worin dieser selbe Professor seine Vorträge als , Warer auf den allgemeinen Markt wirft. Damit tritt Vermehrung des Nationalreichtums ein."

Karl Marx, deutscher Philosoph (1818-1883) 